\title{
Relação entre Medidas Ultra-Sônicas e Espessura de Gordura Subcutânea ou Área de Olho de Lombo na Carcaça em Bovinos de Corte ${ }^{1}$
}

\author{
Jaime Urdapilleta Tarouco², José Fernando Piva Lobato ${ }^{3}$, Adriana Kroef Tarouco ${ }^{4}$, Glauco dos \\ Santos Massia ${ }^{5}$
}

\begin{abstract}
RESUMO - Foram utilizados 162 animais, avaliados em dois anos para determinar a exatidão do ultra-som em estimar a espessura de gordura subcutânea (EGSC) e a área do músculo Longissimus (AOLC) no sítio anatômico entre a $12^{\mathrm{a}}$ e $13^{\mathrm{a}}$ costelas. Dentro de 24 horas antes do abate, foram mensuradas por ultra-som a espessura de gordura subcutânea (EGSUS) e a área de músculo Longissimus (AOLUS). Os coeficientes de correlação simples entre as características medidas por ultra-som e a espessura de gordura subcutânea e área do músculo Longissimus da carcaça foram de 0,95 e 0,97, respectivamente. Diferenças entre medidas ultra-sônicas e da carcaça foram expressas em base atual (EDIFF e ADIFF) e em base absoluta (EDEV e ADEV) para espessura de gordura e área do músculo Longissimus, respectivamente. As médias da EDIFF e ADIFF indicaram que o ultra-som superestima a EGSC em 0,16 mm e subestima a AOLC em 0,26 cm² considerandose ambos os anos. As médias globais da EDEV e ADEV, que são indicações da taxa média de erro, foram 0,34 mm e 1,28 cm², respectivamente. A análise do efeito do ano revelou que EDIFF foi maior no ano 1 e que ADIFF foi maior no ano 2. A análise da EDIFF indicou que animais com EGSC $<2 \mathrm{~mm}$ foram superestimados e com EGSC $>4 \mathrm{~mm}$ serem subestimados pelo ultra-som. Similarmente, houve tendência de animais com AOLC $<50 \mathrm{~cm}^{2}$ foram superestimados e com áreas $>50 \mathrm{~cm}^{2}$ foram subestimados por ultra-som. Os erros-padrão de predição ajustados para viés das medidas ultra-sônicas foram de $0,40 \mathrm{~mm}$ para espessura de gordura subcutânea e de 1,66 $\mathrm{cm}^{2}$ para área do músculo Longissimus. Estes resultados indicam que o ultra-som pode ser um estimador acurado das características de carcaça em bovinos de corte.
\end{abstract}

Palavras-chave: composição corporal, exatidão, ultra-som

\section{Relationship between Ultrasound Measurements and Fat Thickness or Rib Eye Area in Carcass of Beef Cattle}

\begin{abstract}
One hundred sixty-two animals were used during a 2-year period to evaluate the accuracy of ultrasound measurements to estimate carcass subcutaneous fat thickness (CFAT) and area of Longissimus muscle located between the $12^{\text {th }}$ and $13^{\text {th }}$ ribs (CLMA). Twenty-four hours before slaughter animals were ultrasonically measured to determine subcutaneous fat thickness (UFAT) and area of Longissimus muscle (ULMA). The correlation coefficients between ultrasound measurements and CFAT and CLMA were 0.95 and 0.97, respectively. Differences between ultrasonic and carcass measurements were expressed in actual (FDIFF and RDIFF) and absolute (FDEV and RDEV) basis. Means of FDIFF and RDIFF in both years indicated that ultrasound overestimated CFAT by 0,16 mm and underestimated CLMA by $0.26 \mathrm{~cm}^{2}$. Overall means of FDEV and RDEV were $0,34 \mathrm{~mm}$ and $1.28 \mathrm{~cm}^{2}$, respectively. Analysis of year effects showed that FDIFF was greater in year 1 while RDIFF was higher in year 2. FDIFF indicated that ultrasound measurements overestimated CFAT in animals with $<2 \mathrm{~mm}$ CFAT and underestimated CFAT in those with $>4 \mathrm{~mm}$ CFAT. Similarly, animals with CLMA $<50 \mathrm{~cm}^{2}$ were overestimated while those with CLMA $>50 \mathrm{~cm}^{2}$ were underestimated. Standard errors of prediction adjusted for bias of ultrasound measurements were of $0.40 \mathrm{~mm}$ for standard error prediction of subcutaneous fat thickness and $1.66 \mathrm{~cm}^{2}$ for standard error prediction of area of Longissimus muscle. These results suggested that ultrasound can be an accurate estimator of carcass traits in beef cattle.
\end{abstract}

Key Words: accuracy, body composition, ultrasound

\section{Introdução}

A utilização da tecnologia de ultra-sonografia em tempo real para estimar a composição da carcaça in vivo em bovinos não é recente. Essa técnica começou a ser utilizada na década de 50 nos Estados Unidos. Entretanto, no Brasil, os primeiros estudos com bovinos de corte somente foram divulgados na década de 90 .

A variação para características de carcaça dentro dos rebanhos, em que os animais de mesmo sexo, nascidos no mesmo ano e manejados dentro de um mesmo grupo, provavelmente, será pequena. Portanto, um nível muito alto de exatidão das medidas ultra-

\footnotetext{
1 Parte da tese de Doutorado do primeiro autor, financiada pelo CNPq.

2 Zootecnista, Bolsista CNPq (tarouco@fzea.usp.br).

${ }^{3}$ Prof. Adjunto IV, Departamento de Zootecnia/Fac. Agronomia, UFRGS. Bolsista 1 A CNPq. Caixa Postal 15.100, CEP: 90001-970. Porto Alegre-RS-Brasil (jose.fernando.lobato@ufrgs.br).

${ }^{4}$ Médico Veterinário, Bolsista do CNPq (taroucou@terra.com.br).

${ }^{5}$ Médico Veterinário, Autônomo.
} 
sônicas é necessário para classificar os animais com razoável precisão (Robinson et al., 1992).

Segundo Greiner et al. (2003), a variação na exatidão das medidas de ultra-som realizadas pelo mesmo técnico em populações similares de gado, ao longo dos anos, não tem sido investigada. A maioria dos estudos está focada no efeito do animal, do técnico e do equipamento. Entretanto, conforme esses autores, grandes diferenças na exatidão do ultrasom em anos sucessivos têm implicações no progresso genético quando o produtor utiliza esta tecnologia em animais progenitores.

Os objetivos neste estudo foram determinar a relação entre as medidas da área de olho de lombo e espessura de gordura subcutânea, obtidas por ultrasom e na carcaça em populações similares de bovinos, e verificar a exatidão das estimativas da área de olho de lombo e da espessura de gordura subcutânea, obtidas na carcaça e por ultra-som pelo mesmo técnico em anos diferentes.

\section{Material e Métodos}

O estudo foi conduzido em cooperação com os criadores da Conexão Delta G, com sede em Dom Pedrito, RS. Foram utilizados 162 animais da raça Braford, com diferentes graus de sangue, divididos em dois grupos de abate por ano: grupo 1 (Ano 11999) - 102 machos inteiros e 52 machos castrados ao desmame; grupo 2 (Ano 2-2002) - 30 animais machos inteiros e 30 machos castrados ao desmame. Os animais apresentavam média de 12 meses de idade e pertenciam a um mesmo grupo contemporâneo e de manejo dentro de ano. Após o desmame (seis meses) e até o abate, os animais foram mantidos em confinamento e foram alimentados com ração contendo $16 \%$ de proteína bruta (PB) e $81 \%$ de nutrientes digestíveis totais (NDT). Todos os animais receberam identificação única e permanente (brinco e tatuagem na orelha), que foi correlacionada à da carcaça por ocasião do abate, da esfola e da desossa.

A cada ano, os animais foram pesados na fazenda em até 24 horas antes do abate, na mesma data da das medidas ultra-sonográficas (PVUS), considerandose o peso do animal, obtido individualmente em balança eletrônica, após jejum (alimentar e hídrico) de pelo menos 12 horas.

No primeiro ano, as imagens ultra-sônicas foram obtidas utilizando-se uma unidade principal - eco câmera da marca Aloka SSD 500V (Eletro Medicina Berger, Ltda), equipada com um transdutor linear UST 5049 de 3,5MHz de freqüência e com 17,2 cm de comprimento. No segundo ano, uma unidade principal - eco câmera da marca Pie Medical Falcon 100 Vet (Nutricell, Ltda), equipada com um transdutor linear de $3,5 \mathrm{MHz}$ de $18 \mathrm{~cm}$. Para assegurar o contato acústico entre o transdutor e a superfície do sítio anatômico de medida no animal, foi utilizado como acoplante acústico um óleo vegetal. Para melhorar o contato acústico, também foi utilizada uma guia acústica para o ajuste do transdutor à conformação do animal. No sítio anatômico de medida, não foi feito o desbaste do pêlo, realizando-se apenas uma limpeza da área a ser avaliada para evitar interferência da transmissão do feixe de onda sonora.

As medidas ultra-sônicas da área do músculo Longissimus (AOLUS) e da espessura de gordura subcutânea (EGSUS) foram obtidas entre a $12^{\mathrm{a}}$ e $13^{\mathrm{a}}$ costelas, com auxílio da guia acústica. As medidas da AOLUS e EGSUS foram realizadas, respectivamente, delimitando-se a área total do músculo entre o espaço intercostal da $12^{\mathrm{a}}$ e $13^{\mathrm{a}}$ costelas, utilizando-se uma escala de medida em centímetros quadrados $\left(\mathrm{cm}^{2}\right)$. Em todas as medidas, os músculos intercostais (Longissimus costarum) e a depressão de gordura ("acorn fat") na parte proximal do músculo Longissimus foram excluídos.

Para obtenção da espessura de gordura subcutânea, foi medida a profundidade do tecido a $3 / 4$ de distância a partir do lado medial do músculo Longissimus para seu lado lateral, utilizando uma escala em milímetros ( $\mathrm{mm}$ ), utilizando-se um planímetro eletrônico presente no "software" de interpretação de imagens. Após esse procedimento, as imagens obtidas foram armazenadas no disco rígido de um computador portátil e interpretadas a posteriori por meio do "software" ANIMAL ULTRASOUND SERVICE, AUS (AUSKey4W, 1994). Somente uma imagem por animal foi armazenada para cada característica medida por ultra-som. As carcaças foram devidamente identificadas antes de serem submetidas à câmara de resfriamento. A meia-carcaça direita foi utilizada para a obtenção das medidas de área de olho de lombo (AOLC) e da espessura de gordura subcutânea (EGSC) na carcaça.

A espessura de gordura subcutânea (EGSC) utilizada para avaliar a exatidão da medida ultra-sônica foi uma medida não ajustada, obtida a $3 / 4$ de distância 
a partir do lado medial do músculo Longissimus para seu lado lateral, aferida com auxílio de um paquímetro. A área de olho de lombo (AOLC) foi traçada sobre um papel acetato e, posteriormente, medida com uma grade plástica de pontuação AS-234e, distribuída pela Iowa State University of Science and Tecnology, Ames, Iowa, USA (1993).

A análise estatística foi realizada utilizando-se o SAS (2001). Coeficientes de correlação de Pearson foram utilizados para avaliação das relações entre as medidas ultra-sônicas e da carcaça. Análise de variância foi realizada para se verificar diferenças nas características ultra-sônicas e de carcaça entre os dois anos de experimentação. Como nas variáveis de ultra-som e de carcaça, verificou-se diferença significativa a $1 \%$ entre anos apenas para a variável AOLC. Os animais dos dois anos foram considerados em conjunto. A análise de variância também foi realizada para se verificarem diferenças entre abates no ano 1 (1999), constatando-se diferença significativa a $1 \%$ entre abates para a variável EGSC. Assim, todos os animais foram considerados nas análises. Outras variáveis foram criadas para avaliar a exatidão das medidas ultrasônicas em relação às medidas da carcaça (Tabela 1). Foram obtidos, por ultra-som e por medidas na carcaça, o viés e a diferença, em valores absolutos, entre as medidas de espessura de gordura subcutânea (EGS) e da área do músculo Longissimus. As seguintes variáveis específicas foram analisadas:

EDIFF $=($ EGS por ultra-som - EGS na carcaça $)$

$\mathrm{EDEV}=(\mid \mathrm{EGS}$ por ultra-som - EGS na carcaça $\mid)$

$\mathrm{ADIFF}=($ AOLUS $-\mathrm{AOLC})$

$\mathrm{ADEV}=(|\mathrm{AOLUS}-\mathrm{AOLC}|)$

Os termos EDIFF e ADIFF representam a diferença geral média e a direção do viés nas medidas ultrasônicas comparadas com as medidas realizadas na carcaça.

Os termos EDEV e ADEV representam os valores absolutos da diferença entre medidas ultra-sônicas e da carcaça. Análise de variância foi realizada para avaliação das amplitudes específicas das medidas da carcaça, para que a exatidão das medidas ultra-sônicas pudessem ser comparadas dentro das seguintes quatro classes de EGSC: 1) EGSC $\leq 2,0 \mathrm{~mm}$; 2) EGSC $>2,0$ $\mathrm{e} \leq 3,0 \mathrm{~mm}$; 3 ) EGSC $>3,0 \mathrm{e} \leq 4,0 \mathrm{~mm} ; 4)$ EGSC $>4,0$. Similarmente, quatro classes para AOLC foram criadas: 1) AOLC $\leq 50 \mathrm{~cm}^{2}$; 2) AOLC $>50$ e $\leq 55 \mathrm{~cm}^{2}$; 3 ) AOLC $>55 \mathrm{e} \leq 60 \mathrm{~cm}^{2}$; 4) AOLC $>60 \mathrm{~cm}^{2}$.
Tabela 1- Descrição das abreviaturas Table 1 - Abreviation key

\begin{tabular}{lc}
\hline $\begin{array}{l}\text { Abreviatura } \\
\text { Abreviation }\end{array}$ & Definição \\
Definition
\end{tabular}

As descrições das abreviaturas das variáveis encontram-se na Tabela 1.

Foi aplicado o teste de diferença mínima significativa (DMS) para verificar a diferença entre as médias dentro de classes para as variáveis estudadas.

Como o uso dos coeficientes de correlação como medida de acúracia é, muitas vezes, criticado por sua dependência da variância da amostra (Kempster et al., 1982; Houghton \& Turlington, 1992), os erros-padrão de predição (EPP), ajustados para o viés médio nas subclasses de interesse, foram calculados para as variáveis EGSUS e AOLUS. Os EPP são o método estatístico utilizado para avaliar a exatidão do técnico de ultra-som e são medidos como o desvio-padrão da diferença entre medidas ultra-sônicas e da carcaça para um dado técnico. O EPP é a medida do grau de variabilidade, ajustado para o viés, que ocorre na predição das medidas da carcaça a partir de medidas ultra-sônicas. Essa medida pri- 
mária avalia a habilidade do técnico em classificar ou predizer diferenças entre animais corretamente (Wilson, 1999).

\section{Resultados e Discussão}

A média e a amplitude do PCQ (Tabela 2) foram de 157,29 e $87,80 \mathrm{~kg}$, respectivamente, para todo o conjunto de dados.

Para a EGSC e AOLC, considerando-se os dados dos dois anos em conjunto (Tabela 2), a média foi de $3,65 \mathrm{~mm}$, com desvios-padrão de $1,24 \mathrm{~mm}$ e de 53,74 $\mathrm{cm}^{2}$ e $6,05 \mathrm{~cm}^{2}$, respectivamente.

Os coeficientes de correlação entre as medidas ultra-sônicas e de carcaça (Tabela 3) para a espessura de gordura subcutânea $(0,94)$ e área de músculo $(0,96)$ são similares aos obtidos por Perry et al. (1990), Bullock et al. (1991), Brethour (1992), Bergen et al. (1996) e Greiner et al. (2003), que obtiveram coeficientes de 0,$96 ; 0,90 ; 0,90$ e 0,92 para área de músculo e 0,$90 ; 0,90 ; 0,91$ e 0,92 para espessura de gordura, respectivamente.

Tabela 2 - Médias, desvios-padrão e amplitudes de medidas da carcaça e ultra-som $(n=162)^{1}$

Table 2 - Means, standard deviations and range of carcass and ultrasound measurements $(n=162)^{1}$

\begin{tabular}{|c|c|c|c|}
\hline $\begin{array}{l}\text { Característica } \\
\text { Trait }\end{array}$ & $\begin{array}{l}\text { Média } \\
\text { Mean }\end{array}$ & $\begin{array}{l}\text { Desvio-padrão } \\
\text { Standard deviation }\end{array}$ & $\begin{array}{l}\text { Amplitude } \\
\text { Range }\end{array}$ \\
\hline $\begin{array}{l}\text { PVUS, kg } \\
\text { FWT, kg }\end{array}$ & 295,90 & 26,81 & 134,00 \\
\hline $\begin{array}{l}\text { PCQ, kg } \\
\mathrm{HCW}, \mathrm{kg}\end{array}$ & 157,29 & 14,71 & 87,80 \\
\hline $\begin{array}{l}\text { EGSU,mm } \\
\text { UFAT,mm }\end{array}$ & 3,80 & 1,11 & 6,00 \\
\hline $\begin{array}{l}\text { AOLU, } \mathrm{cm}^{2} \\
\text { ULMA, } \mathrm{cm}^{2}\end{array}$ & 53,48 & 5,50 & 26,64 \\
\hline $\begin{array}{l}\text { EGSC,mm } \\
\text { CFAT, mm }\end{array}$ & 3,65 & 1,24 & 6,50 \\
\hline $\begin{array}{l}\text { AOLC, } \mathrm{cm}^{2} \\
\text { CLMA, } \mathrm{cm}^{2}\end{array}$ & 53,74 & 6,05 & 33,54 \\
\hline $\begin{array}{l}\text { EDIFF,mm } \\
\text { FDIFF,mm }\end{array}$ & $0,16^{* *}$ & 0,40 & 2,20 \\
\hline $\begin{array}{l}\text { EDEV,mm } \\
\text { FDEV,mm }\end{array}$ & 0,34 & 0,25 & 1,20 \\
\hline $\begin{array}{l}\text { ADIFF, } \mathrm{cm}^{2} \\
\text { RDIFF }, \mathrm{cm}^{2}\end{array}$ & $-0,26$ & 1,66 & 12,57 \\
\hline $\begin{array}{l}\text { ADEV }, \mathrm{cm}^{2} \\
\text { RDEV }, \mathrm{cm}^{2}\end{array}$ & 1,28 & 1,09 & 7,99 \\
\hline
\end{tabular}

1 Ver Tabela 1 para descrição das abreviaturas.

1 See Table 1 for abreviations key.

** Médias diferem a partir de zero $(P<0,001)$.

** Means values different from zero $(P<0.001)$.
A alta relação encontrada neste estudo pode ser atribuída vários fatores: 1) a experiência do técnico: vários autores afirmam que a repetibilidade e a exatidão das medidas ultra-sônicas aumentam com a experiência (Wallace \& Stouffer, 1974; Herring et al., 1994; Hassen et al., 1998); 2) a utilização de um computador e de um software específico na coleta e interpretação das imagens ultra-sônicas, que, segundo Robinson et al. (1992), melhora em 10\% a exatidão da medida de área de músculo; 3 ) o fato de todos os animais avaliados apresentarem espessura de gordura de cobertura menor que $10 \mathrm{~mm}$, possibilitando o estreitamento dos valores da mesma medida obtida na carcaça.

Conforme Wilson et al. (1999), em animais gordos (>12 mm), há maior dificuldade na obtenção de uma imagem clara e de alta qualidade, diminuindo a exatidão das medidas musculares.

Neste estudo, os animais apresentavam gordura de cobertura entre 1,8 e $7,8 \mathrm{~mm}$, com média de 3,8 $\mathrm{mm}$, o que pode ter influenciado a qualidade das imagens e, conseqüentemente, a exatidão, estreitando a relação entre as características avaliadas. O tamanho da área do músculo também pode ser considerado uma fonte de variação na relação entre as medidas ultra-sônicas e na carcaça. Conforme Perkins et al. (1992a), à medida que as áreas de olho de lombo dos animais aumentaram, a correlação diminuiu. Segundo Wilson (1999), Duello (1993) e Greiner et al. (2003), há uma tendência de as áreas musculares menores serem superestimadas e as maiores serem subestima-

Tabela 3 - Coeficientes de correlação entre as medidas de carcaça e ultra-som $(n=162)^{1}$

Table 3 - Correlation coefficients between ultrasound and carcass measurements $(n=162)^{1}$

\begin{tabular}{lcccc}
\hline $\begin{array}{l}\text { Característica } \\
\text { Trait }\end{array}$ & $\begin{array}{c}\text { EGSUS } \\
\text { UFAT }\end{array}$ & $\begin{array}{c}\text { AOLUS } \\
\text { ULMA }\end{array}$ & $\begin{array}{c}\text { EGSC } \\
\text { CFAT }\end{array}$ & $\begin{array}{c}\text { AOLC } \\
\text { CLMA }\end{array}$ \\
\hline EGSUS & - & $-0,037$ & $0,948^{* *}$ & $-0,08$ \\
UFAT & & - & $-0,057$ & $0,963^{* *}$ \\
AOLUS & & - & & \\
ULMA & & & - & 0,101 \\
EGSC & & & & - \\
CFAT & & & & - \\
AOLC & & & & \\
CLMA & & &
\end{tabular}

1 Ver Tabela 1 para descrição das abreviaturas.

1 See Table 1 for abreviations key.

** Coeficientes de correlação diferem a partir de zero $(P<0,001)$.

** Correlation coefficients different from zero $(P<0.001)$. 
das pela técnica de ultra-som. $\mathrm{O}$ avaliador que realiza a medição da área de músculo da carcaça pode influenciar na relação entre as características (Robinson et al., 1992; Rouse et al., 1993). O método de medida feito na carcaça também influencia sua repetibilidade. Steiner et al. (2003) obtiveram diferença média absoluta de $1,29 \mathrm{~cm}^{2}$ entre medidas individuais utilizando a grade de pontuação diretamente no sítio de medida na carcaça e de $1,35 \mathrm{~cm}^{2}$ coletando com papel acetato e medindo a posteriori com planímetro. Outra fonte de variação é o manejo da carcaça após o abate. Segundo Brethour (1992), quando a remoção do couro é mecânica, retiram-se pedaços de gordura aderidos ao couro ou separam-se as camadas de gordura da carcaça.

A área média de músculo $\left(53,48 \mathrm{~cm}^{2}\right)$ registrada encontra-se na faixa aceitável para boa resolução de imagem e alta precisão. A análise do viés médio (EDIFF) revelou que o ultra-som superestimou a espessura de gordura da carcaça em 0,16 mm (Tabela 2) e essa diferença é significativamente diferente de zero $(\mathrm{t}=5,01 ; \mathrm{P}<0,0001)$. Resultados semelhantes foram obtidos por diversos autores, que afirmam que, quando a espessura de gordura subcutânea é menor que $8 \mathrm{~mm}$, o ultra-som tende a superestimar a gordura na carcaça (Herring et al.,1994; Hassen et al.,1998; Charagu et al., 2000; Greiner et al., 2003).

Neste estudo, os animais apresentavam espessura menor que $6 \mathrm{~mm}$, com média de $3,8 \mathrm{~mm}$. A média da EDEV indicou diferença absoluta média de $0,34 \mathrm{~mm}$ entre a EGSUS e EGSC. Esse valor é inferior aos resultados obtidos por Duello (1992), Perkins et al.(1992b), Charagu et al. (2000) e Greiner et al. (2003), de 2,3; 1,9; 2,2 e 1,38 mm, respectivamente.

Segundo Robinson et al. (1992), esses resultados poderiam ser, em parte, justificados pela relutância dos técnicos em reportar medidas entre 0 e $1 \mathrm{~mm}$, ou talvez, pela convenção de tomar medidas a partir da parte superior da banda, indicando os limites entre as camadas da gordura e o músculo. Esses autores comentam que, embora a linha superior que separa o tecido represente o limite das camadas de gordura subcutânea, a profundidade das fáscias é mais distinguível em animais mais gordos. Neste caso, os valores obtidos foram mais baixos, podendo ser atribuídos à familiaridade em avaliar animais com menor grau de acabamento. As divergências nos resultados podem ser, em parte, decorrentes do erro do técnico na coleta, na interpretação das imagens, na calibração do equipamento, pois, segundo Brethour (1992), não se faz a correção da velocidade do som na gordura dos bovinos, que é $7 \%$ mais baixa que a velocidade calibrada no equipamento $(1540 \mathrm{~m} / \mathrm{s})$.

A medida de ultra-som subestimou a área de músculo (ADIFF) em aproximadamente $0,26 \mathrm{~cm}^{2}$, com um desvio-padrão de $1,66 \mathrm{~cm}^{2}$ (Tabela 2), de modo que ADIFF não diferiu significativamente de zero $(\mathrm{t}=-1,98 ; \mathrm{P}=0,0489)$. A mesma tendência foi observada por Perkins et al. (1992a), de $1,7 \mathrm{~cm}^{2} \mathrm{em}$ novilhos, e Crews et al. (2002), de 4,25; 5,66 e $1,23 \mathrm{~cm}^{2}$ em novilhos, touros e novilhas, respectivamente. Esses resultados confirmam os descritos por May et al. (2000), que constataram que a maioria das observações $(64,7 \%)$ foram subestimadas.

A diferença média absoluta (ADEV) entre AOLUS e AOLC foi de $1,28 \mathrm{~cm}^{2}$, com um desviopadrão de 1,09 $\mathrm{cm}^{2}$ (Tabela 2). Entretanto, foi mais exata a obtida por Herring et al. (1994), de 4,94 a $6,76 \mathrm{~cm}^{2}$, e Greiner et al. (2003), de 3,31 cm $\mathrm{cm}^{2}$, ambos trabalhando com um equipamento idêntico ao utilizado no primeiro ano deste experimento.

A análise de variância revelou que o ano pode ser uma significativa fonte de variação para as variáveis medidas por ultra-som (Tabela 4).

As médias de EDIFF e EDEV apresentaram maior magnitude no ano 1 . O ultra-som superestimou a espessura de gordura subcutânea em aproximadamente $0,20 \mathrm{~mm}$ e essa diferença diferiu significativamente de zero $(t=4,99, P<0,0001)$, enquanto, entre AOLUS e AOLC, essa diferença $\left(0,15 \mathrm{~cm}^{2}\right)$ não diferiu significativamente de zero $(\mathrm{t}=0,96$, $\mathrm{P}=0,3414$ ) para o ano 1 . Isso foi confirmado pelos valores pequenos das características ADIFF $\left(0,15 \mathrm{~cm}^{2}\right)$ e $\operatorname{ADEV}\left(1,11 \mathrm{~cm}^{2}\right)$. Resultados similares para ADIFF foram obtidas por Greiner et al. (2003), 0,55 $\mathrm{cm}^{2}$, em animais com áreas de 77,4 a $83,9 \mathrm{~cm}^{2}$ e com a mesma tendência, porém, com valores superiores; e por Perkins et al. (1992 b), em novilhos cruzados $\left(1,1 \mathrm{~cm}^{2}\right)$.

Quanto à variável ADEV, valores superiores foram obtidos por Crews et al. (2002) de 4,96; 5,96 e $3,32 \mathrm{~cm}^{2}$, em novilhos, touros e novilhas, respectivamente, e por Greiner et al. (2003), 2,87 $\mathrm{cm}^{2}$, em novilhos com menos de $5,1 \mathrm{~mm}$ de espessura de gordura de cobertura.

Os valores mais elevados foram encontrados por Crews et al. (2002) e Greiner et al. (2003) revelam maior variação nas características em relação às 
Tabela 4 - Médias e desvios-padrão (DP) para as medidas ultra-sônicas e da carcaça por ano ${ }^{1}$ Table 4 - Means and standard deviations (SD) of carcass and ultrasound measurements in each year ${ }^{1}$

\begin{tabular}{|c|c|c|c|c|}
\hline \multirow[b]{2}{*}{$\begin{array}{l}\text { Característica } \\
\text { Trait }\end{array}$} & \multicolumn{2}{|c|}{$\begin{array}{l}\text { Ano } 1(\mathrm{n}=102) \\
\text { Year } 1(n=102)\end{array}$} & \multicolumn{2}{|c|}{$\begin{array}{l}\text { Ano } 2(n=60) \\
\text { Year } 2(n=60)\end{array}$} \\
\hline & $\begin{array}{l}\text { Média } \\
\text { Mean }\end{array}$ & $\begin{array}{l}\mathrm{DP} \\
S D\end{array}$ & $\begin{array}{l}\text { Média } \\
\text { Mean }\end{array}$ & $\begin{array}{l}\text { DP } \\
S D\end{array}$ \\
\hline $\begin{array}{l}\mathrm{PCQ}, \mathrm{kg} \\
\mathrm{HCW}, \mathrm{kg}\end{array}$ & 158,25 & 16,28 & 155,65 & 11,51 \\
\hline $\begin{array}{l}\text { EGSUS, } \mathrm{mm} \\
U F A T, \mathrm{~mm}\end{array}$ & 3,94 & 1,23 & 3,58 & 0,83 \\
\hline $\begin{array}{l}\text { AOLUS, } \mathrm{cm}^{2} \\
U L M A, \mathrm{~cm}^{2}\end{array}$ & 52,68 & 5,29 & 54,84 & 5,63 \\
\hline $\begin{array}{l}\mathrm{EGSC}, \mathrm{mm} \\
C F A T, m m\end{array}$ & 3,73 & 1,35 & 3,50 & 1,03 \\
\hline $\begin{array}{l}\text { AOLC }, \mathrm{cm}^{2} \\
C L M A, \mathrm{~cm}^{2}\end{array}$ & 52,53 & 5,51 & 55,79 & 6,42 \\
\hline $\begin{array}{l}\text { EDIFF, mm } \\
F D I F F, m m\end{array}$ & $0,20 * *$ & 0,41 & 0,08 & 0,36 \\
\hline $\begin{array}{l}\mathrm{EDEV}, \mathrm{mm} \\
F D E V, \mathrm{~mm}\end{array}$ & 0,37 & 0,27 & 0,29 & 0,21 \\
\hline $\begin{array}{l}\text { ADIFF, } \mathrm{cm}^{2} \\
R D I F F, \mathrm{~cm}^{2}\end{array}$ & 0,15 & 1,54 & $-0,95 * *$ & 1,64 \\
\hline $\begin{array}{l}\mathrm{ADEV}, \mathrm{cm}^{2} \\
R D E V, \mathrm{~cm}^{2}\end{array}$ & 1,11 & 1,08 & 1,57 & 1,05 \\
\hline
\end{tabular}

deste estudo, influenciando a magnitude da exatidão. Isso se deve, em parte, à diferença dos tipos biológicos dos animais estudados, diferentemente deste estudo, em que os animais pertenciam ao mesmo tipo biológico, mesmo grupo contemporâneo e foram submetidos ao mesmo manejo e nível nutricional desde o nascimento.

Vários pesquisadores têm demonstrado que a magnitude das diferenças entre EGSUS e EGSC aumenta quando a espessura de gordura subcutânea na carcaça se eleva (Brethour, 1992; Duello, 1992, Herring et al., 1994), o que foi confirmado pelo valor médio da característica $\operatorname{EDEV}(0,37 \mathrm{~mm})$. Entretanto, as correlações entre EGSUS e EGSC foram iguais ( $\mathrm{r}=$ 0,952, ano 1 e 0,948, ano 2), o que reforça a afirmação de Herring et al. (1994) de que deve-se ter cuidado ao interpretar os coeficientes de correlação e, quando possível, utilizar outro método estatístico para confirmar a repetibilidade e exatidão das medidas. Hougthon $\&$ Turlington (1992) sugerem que a magnitude das correlações é muito dependente da variação populacional e não reflete o viés das características.

Resultados similares foram reportados por Greiner et al. (2003), ao utilizarem a mesma metodologia deste estudo. Eles reportam que, apesar de a diferença entre as médias de EGSUS e EGSC entre anos ter sido considerada pequena $(1 \mathrm{~mm})$ e a diferença entre os desvios-padrão não terem sido elevadas $(0,5 \mathrm{~mm})$, as diferenças entre as médias de EDIFF foram significativas ( $\mathrm{P}<0,001)$, assim como os coeficientes de correlação obtidos entre EGSUS e EGSC foram similares $(\mathrm{r}=0,86$ e 0,90$)$ para os anos 1 e 2 , respectivamente.

O ultra-som, no ano 2, subestimou a área de olho de lombo em aproximadamente $0,95 \mathrm{~cm}^{2}$ (ADIFF) e essa diferença foi significativamente diferente de zero ( $\mathrm{t}=-4,47, \mathrm{P}<0,0001)$, ao passo que, entre as médias EGSUS e EGSC, a diferença foi de $0,08 \mathrm{~mm}$ e não diferiu significativamente de zero $(\mathrm{t}=1,63$, $\mathrm{P}=0,1079)$. Essa não-significância pode ser confirmada pela diminuição da EDEV do segundo ano $(0,2 \mathrm{~mm}) \mathrm{em}$ comparação à do primeiro ano $(0,37 \mathrm{~mm})$.

Segundo Hassen et al. (1998), durante o processo de computar médias absolutas (isto é, viés médio absoluto), ocorre total cancelamento dos desvios negativos e positivos e, conseqüentemente, um viés médio próximo de zero pode, não necessariamente, implicar em uma medida acurada. Entretanto, quando se utiliza o viés em módulo (EDIFF), evita-se esse problema de anulação dos desvios, que pode ser confir- 
mado pela diferença entre os valores obtidos entre os anos para esta variável (Tabela 4 , EDIFF ano $1=$ 0,20 mm, $\mathrm{P}<0,001$; EDIFF ano $2=0,08 \mathrm{~mm}$ ), o que significa que a estimativa da EGSC por ultra-som no segundo ano foi mais exata que no ano 1 .

As médias para as características ADIFF $\left(-0,95 \mathrm{~cm}^{2}\right)$ e $\operatorname{ADEV}\left(1,57 \mathrm{~cm}^{2}\right)$ foram maiores em magnitude no ano 2. A diferença em relação ao ano 1 pode ser, em parte, explicada pelo aumento do ADEV de $1,11 \mathrm{~cm}^{2}$ no primeiro ano para $1,57 \mathrm{~cm}^{2}$ no segundo, indicando que a estimativa da AOLC pelo ultra-som foi mais exata no ano 1, apesar de apresentar coeficiente de correlação mais elevado que o do ano $2(\mathrm{r}=0,97)$. A explicação para esse resultado provavelmente está na variação apresentada por essa característica no ano 2 . A AOLC no ano 1 apresentou amplitude de $33,54 \mathrm{~cm}^{2}$, que foi mais elevada que a de $25,80 \mathrm{~cm}^{2}$ do primeiro ano. Esses resultados são confirmados pela afirmativa de Kempster et al. (1982) de que o coeficiente de correlação é influenciado pela amplitude dos valores da amostra em que está baseado. Embora satisfatório para comparar preditores na mesma amostra de carcaças, o coeficiente de correlação não é adequado para a comparação da precisão quando os dados de carcaça são obtidos em diferentes amostras.

Segundo Greiner et al. (2003), as medidas da carcaça não são obtidas sem que erros sejam cometidos e isto poderia, também, afetar os resultados sobre a exatidão do ultra-som. Robinson et al. (1992) e Steiner et al (2003) descrevem bem esse fato, reportando diferenças de até $1,87 \mathrm{~cm}^{2}$ entre medidas repetidas na mesma carcaça, por um mesmo avaliador, em um mesmo sítio anatômico, provavelmente em razão dos desvios na identificação dos limites do músculo.

Os EPP para as características estudadas encontram-se na Tabela 5. Os EPP foram primeiramente propostos por Robinson et al. (1992) para medir o grau de variabilidade que ocorria na predição de medidas da carcaça a partir de medidas ultra-sônicas, por considerar o viés do técnico ou do equipamento, uma vez que cada medida é desviada de sua média (Herring et al., 1994). Segundo Robinson et al. (1992), a escolha foi feita visando substituir a média absoluta das diferenças, em razão de sua aceitação geral como medida de variabilidade, e porque, quando as diferenças são elevadas ao quadrado, um pequeno número de erros grandes é considerado mais importante que um grande número de pequenas discrepâncias. Esse pro-

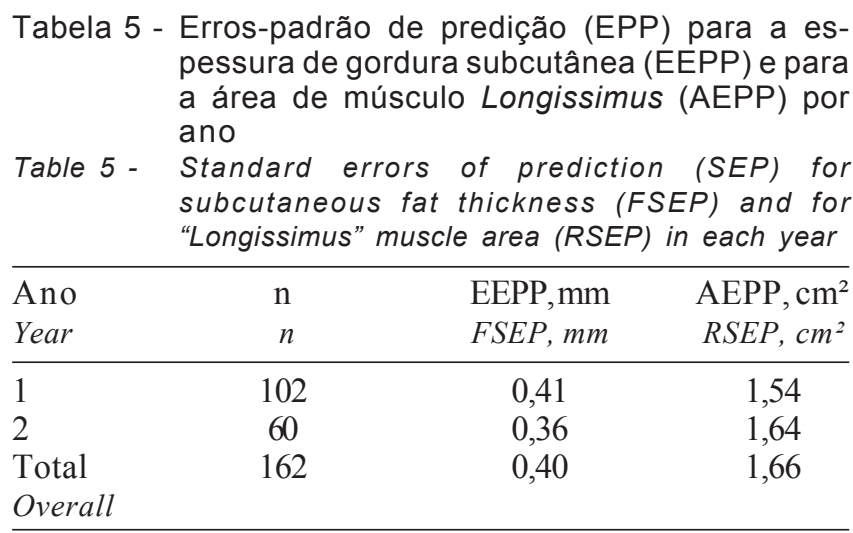

cedimento foi considerado importante no estudo, porque, no Brasil, ainda não se têm estimativas de parâmetros estatísticos da exatidão desta tecnologia para certificar os técnicos que a utilizam em programas de seleção genética para as características de carcaça em animais de tipos biológicos criados nos sistemas brasileiros de produção.

O erro-padrão de predição para espessura de gordura subcutânea (EEPP) foi similar entre anos, embora maior viés tenha sido verificado no primeiro ano, como pode ser observado pelos valores da EDIFF (Tabela 4). Desse modo, após a correção para os vieses, os dados sugerem que a exatidão do ultra-som em medir a EGSC foi similar para ambos os anos (Tabela 6).

Os resultados deste estudo são inferiores aos obtidos por Robinson et al. (1992) para espessura de gordura; o EEPP por ultra-som (Aloka DX210 e Toshiba SAL 32B) variou de 0,67 a 1,26 mm para diferentes técnicos com três dias de aperfeiçoamento na utilização da tecnologia, trabalhando com animais com média de 4,5 $\mathrm{mm}$ de espessura de gordura subcutânea na carcaça. Crews et al. (2002) reportaram valores de EEPP por ultra-som (SSD-Aloka 1100 Flexus) para a EGSC de 0,87 a 1,51 mm, em animais cuja média de espessura de gordura na carcaça era de 13,$19 ; 4,52$ e 7,76 mm para novilhos, touros e novilhas, respectivamente, sendo a medida ultra-sônica coletada dentro de um intervalo de 3 a 7 dias.

Greiner etal.(2003) obtiveram EEPP de 1,9 e 2,1 mm para a mesma característica, para os anos 1 e 2, respectivamente, em animais de EGSC de 10,2 mm, em média, destacando-se que a medida ultra-sônica foi coletada (Aloka SSD $500 \mathrm{~V}$ ) cinco dias antes do abate. O melhor resultado obtido neste estudo pode ser atribuído ao tipo de equipamento, ao tempo transcorrido da medida ultra-sônica ao abate (24 horas), ao 
grau de acabamento dos animais utilizados na experimentação e à experiência do técnico em avaliar animais com menor padrão de acabamento.

Em contraste à característica de espessura de gordura, os dados para a área do músculo foram menos viesados no primeiro ano (consultar valores ADIFF; Tabela 4), refletindo em um valor mais elevado do erro-padrão de predição para área do músculo Longissimus (AEPP) no segundo ano. O valor do AEPP sugere que AOLC foi mais acuradamente predita no primeiro ano. Robinson et al. (1992) obtiveram valores do AEPP de 5,0 $\mathrm{cm}^{2}$ na estimativa da AOLC por ultra-som. Crews et al.(2002) obtiveram valores do AEPP de 4,49; 4,75 e 4,93 $\mathrm{cm}^{2}$ para novilhos, novilhas e touros, respectivamente, na estimativa da AOLC por ultra-som.

Conforme Greiner et al. (2003), o baixo AEPP obtido indica que o ultra-som pode ser usado para, acuradamente, identificar diferenças em AOLC entre animais. O AEPP médio deste estudo foi de $1,66 \mathrm{~cm}^{2}$ e pode ser decorrente do tamanho dos animais utilizados no experimento, diminuindo as fontes que influenciam nas inconsistências na coleta e interpretação das imagens ultra-sônicas. A correta localização e visualização da porção medial do músculo longissimus é afetada pela quantidade de eco que retorna ao transdutor. Este retorno é menor em animais com alto grau de acabamento e/ou musculatura muito desenvolvida, em razão da maior profundidade que a onda sonora precisa penetrar para a visualização dos limites teciduais. Com isso, há maior atenuação do feixe sonoro em virtude da dispersão e absorção pelo calor, acarretando menor resolução da imagem quando o tecido a ser avaliado está a uma maior profundidade. Conforme Cross (1989), o discernimento dos limites do músculo Longissimus é problemático nos equipamentos de ultra-som em tempo real, podendo ser explicado pela refração das ondas sonoras na curvatura final do músculo e pelo aumento da atenuação em tecidos profundos. Segundo Perkins (1992), o ultra-som estima mais precisamente a AOLC de animais com áreas de músculos pequenas e a exatidão é mais influenciada pelo grau de acabamento e musculosidade dos animais que pela experiência do técnico. Esta tendência pode ser confirmada, em parte, pelos resultados deste trabalho, em que o valor do AEPP (Tabela 8) aumentou à medida que a área de músculo excedeu os $50 \mathrm{~cm}^{2}$.

Com a finalidade de avaliar as diferenças em acuracidade do ultra-som, como resultado da magni- tude das medidas da carcaça, os dados foram divididos em quatro classes baseadas nas características de EGSC e AOLC.

Os quadrados médios mínimos e erros-padrão da exatidão das variáveis EGSC e AOLC dentro de categorias encontram-se nas Tabelas 7 e 8, respectivamente. As médias de EDIFF por EGSC categórico indicam que a espessura de gordura da carcaça tem efeito sobre o erro das medições de AOLU e EGSU; animais mais magros ( $<4 \mathrm{~mm}$ EGSC) foram superestimados e aqueles com mais de $4 \mathrm{~mm}$ de EGSC foram subestimados utilizando-se o ultrasom (Tabela 7). Para as classes de espessura de gordura na carcaça, foram encontradas diferenças significativas para EDIFF, EDEV e ADIFF. Os quadrados médios mínimos da EDEV por categoria de EGSC sugerem que as diferenças absolutas entre EGSUS e EGSC foram similares nas categorias extremas de gordura $(<2 \mathrm{~mm}$ e $>4 \mathrm{~mm})$; as categorias de 2-3 e 3-4 mm também foram similares, assim como as categorias de $>4 \mathrm{~mm}$ e $2-3 \mathrm{~mm}$. Houve diferença significativa $(\mathrm{P}<0,05)$ entre as categorias $<2 \mathrm{~mm}$ e 2-3 $\mathrm{mm}$ e entre as categorias 3$4 \mathrm{~mm}$ e $>4 \mathrm{~mm}$, o que quer dizer que as diferenças absolutas são maiores nas categorias de $<2 \mathrm{~mm} \mathrm{e}$ $>4 \mathrm{~mm}$ e que o ultra-som foi mais exato na sua predição quando os animais apresentavam espessura de gordura subcutânea de 3 a $4 \mathrm{~mm}$, que é confirmado pelo valor mais baixo da $\operatorname{EDIFF}(0,11)$ nesta categoria.

Os resultados deste trabalho estão de acordo, em parte, com os de Greiner et al. (2003), que encontraram a mesma tendência nas categorias de animais magros $(<5,1 \mathrm{~mm})$, nas quais o ultra-som superestimou, e de animais gordos $(>7,6 \mathrm{~mm})$, nas quais subestimou a espessura de gordura na carcaça.

A mesma tendência foi obtida por Herring et al. (1994), que relataram que o técnico também poderia ser responsável pela resposta de superestimar a EGSC e a AOLC em novilhos pouco musculosos e magros em superestimar as mesmas características em novilhos de alta musculosidade e maior grau de acabamento quando obtidas por ultra-som.

Os resultados podem ser explicados, em parte, pela constatação de Robinson et al. (1992) de que, pelo fato de o sítio anatômico entre a $12^{\mathrm{a}}$ e $13^{\mathrm{a}}$ costelas estar localizado sobre uma área côncava, a suspensão da carcaça na nória faz com que as camadas de gordura se agrupem neste ponto, aumentando a espessura de 
Tabela 6 - Quadrados médios mínimos e erros-padrão (EP) da exatidão das variáveis pelo EGSC categórico ( $\mathrm{n}=162$ ) Table 6 - Accuracy of least square means and standard errors (SD) of different variables in each CFAT category ( $n=162)$

\begin{tabular}{|c|c|c|c|c|c|}
\hline $\begin{array}{l}\text { EGSC categórico } \\
C F A T \text { category }\end{array}$ & $\begin{array}{l}\mathrm{n} \\
n\end{array}$ & $\begin{array}{l}\text { EDIFF,mm } \\
F D I F F, m m\end{array}$ & $\begin{array}{l}\mathrm{EDEV}, \mathrm{mm} \\
F D E V, m m\end{array}$ & $\begin{array}{l}\text { ADIFF,cm } \\
R D I F F, \mathrm{~cm}^{2}\end{array}$ & $\begin{array}{l}\mathrm{ADEV}, \mathrm{cm}^{2} \\
R D E V, \mathrm{~cm}^{2}\end{array}$ \\
\hline $\begin{array}{l}<2 \mathrm{~mm} \\
2-3 \mathrm{~mm} \\
3-4 \mathrm{~mm} \\
>4 \mathrm{~mm}\end{array}$ & $\begin{array}{l}19 \\
51 \\
51 \\
41\end{array}$ & $\begin{array}{r}0,44^{b_{ \pm}} \pm 0,08 \\
0,30^{b_{ \pm}} \pm 0,04 \\
0,11^{c_{ \pm}} \pm 0,05 \\
-0,10^{d_{ \pm 0,07}}\end{array}$ & $\begin{array}{l}0,48^{\mathrm{b}_{ \pm 0}} 07 \\
0,36^{\mathrm{cd}} \pm 0,03 \\
0,25^{\mathrm{d}_{ \pm}} \pm 0,03 \\
0,38^{\mathrm{bc}_{ \pm 0,04}}\end{array}$ & $\begin{array}{c}-0,71^{\mathrm{cd}_{ \pm 0,28}} \\
-0,91^{\mathrm{d}_{ \pm 0}}, 21 \\
0,46^{\mathrm{b}_{ \pm 0,26}} \\
-0,14^{\mathrm{bc}_{ \pm 0,21}}\end{array}$ & $\begin{array}{l}1,20 \pm 0,17 \\
1,36 \pm 0,16 \\
1,32 \pm 0,19 \\
1,17 \pm 0,11\end{array}$ \\
\hline
\end{tabular}

1 Ver Tabela 1 para descrição das abreviaturas.

1 See Table 1 for abreviations key.

a,b,c,d Médias seguidas de letras distintas na coluna diferem significativamente $(P<0,05)$ pelo teste DMS.

$a, b, c, d$ Means in a column with different superscripts differ $(P<0.001) L S D$ test.

gordura subcutânea, se comparada à de animais em posição ereta, ressaltando-se que este efeito é maior em animais com maior grau de acabamento.

Com a mesma tendência dos resultados de Greiner et al. (2003), que encontraram animais com menos de 5,1 mm de EGSC, o ultra-som subestima a AOLC. Os resultados deste trabalho, pelos valores e pelo sinal negativo da ADIFF (Tabela 7), demonstram que até 3 mm de EGSC, a área de músculo é subestimada. Entretanto, a maior exatidão na estimativa da AOLC por ultra-som ocorreu na categoria de $>4 \mathrm{~mm}$, podendo ser confirmada pelo valor da ADIFF $\left(-0,14 \mathrm{~cm}^{2}\right)$. $\mathrm{O}$ resultado neste caso decorreu, provavelmente, do fato de que animais com maior espessura de cobertura de gordura apresentavam, geralmente, uma conformação mais convexa, o que facilitou o maior contato entre o transdutor e a região a ser avaliada. Com isso, foi obtida uma imagem de alta resolução em comparação à de animais mais magros, de conformações mais côncavas, que dificultaram o contato da área de medida com a sonda ultra-sônica.

Para as classes de área de músculo, verificaramse diferenças significativas para a ADIFF e ADEV (Tabela 7). Os resultados demonstram que animais com AOLC menores que $50 \mathrm{~cm}^{2}$ são superestimados por ultra-som e que aqueles com áreas maiores que 50 $\mathrm{cm}^{2}$ de AOLC são subestimados quando a estimativa é realizada por ultra-som. A média de AOLC categórico $\left(<50 \mathrm{~cm}^{2}\right)$ diferiu significativamente $(\mathrm{P}<0,005)$ das demais, não havendo diferença significativa entre as médias do AOLC categórico (50-55 $\left.\mathrm{cm}^{2}\right)$ e da categoria de AOLC de áreas entre 55 e $60 \mathrm{~cm}^{2}$. A média do AOLC categórico $\left(50-55 \mathrm{~cm}^{2}\right)$ não diferiu significativamente da média da categoria de AOLC de $>$ de $60 \mathrm{~cm}^{2}$. A média da ADIFF da classe de AOLC entre 50 e $55 \mathrm{~cm}^{2}$ indica uma tendência de maior exatidão de estimativa por ultra-som nesta amplitude de área, estando confirmada pelos valores menores para esta classe da ADEV e do EPP, de $0,95 \pm 0,10$, respectivamente (Tabela 7). Observou-se ainda, que os valores do viés absoluto são maiores nos animais com pequenas áreas de músculo $\left(<50 \mathrm{~cm}^{2}\right) \mathrm{e}$ nos de grandes AOLC categórico $\left(>60 \mathrm{~cm}^{2}\right)$. Estes valores estão de acordo com as expectativas estatísticas e com estudos prévios que examinaram o viés das medidas ultra-sônicas em diferentes categorias de EGSC e AOLC (Smith et al., 1992; Herring et al., 1994; Greiner et al., 2003).

Os EPP para a EGSC e AOLC por categoria estão apresentados na Tabela 8 . Os resultados estão de acordo com as variáveis DIFF e DEV discutidas previamente. As predições mais exatas para a espessura de gordura subcutânea da carcaça por ultra-som foram localizadas nas classes intermediárias do EGSC categórico, ou seja, de 2 a $4 \mathrm{~mm}$ e as menos exatas nas classes extremas $(<2 \mathrm{~mm}$ e $>4 \mathrm{~mm})$.

Os resultados para a classe de gordura $<2 \mathrm{~mm}$ podem ser confirmados pela afirmação de Brethour (1992) de que os limites das camadas mais profundas da gordura no ultra-som são menos aparentes em animais magros e mais distinguíveis em animais com maior grau de acabamento e, conseqüentemente, mais correlatas com a espessura da carcaça. $\mathrm{Na}$ classe de EGSC $>4 \mathrm{~mm}$, a fonte de variação pode ser decorrente da pressão exercida pelo técnico no transdutor durante a coleta da imagem, podendo diminuir a espessura de gordura, principalmente em animais mais gordos, o que pode ser confirmado pelo valor da EDIFF (-0,10, Tabela 6). Segundo Heckmatt et al. (1988), a quantidade de pressão aplicada ao transdutor durante o procedimento de coleta da imagem ultra-sônica pode causar distorções no tecido e afetar a exatidão das medidas de espessura de gordura subcutânea, porque 
Tabela 7 - Quadrados médios mínimos* e erros-padrão $(E P)$ da exatidão das variáveis pelo $A O L C$ categórico $^{1}(\mathrm{n}=162)$ Table 7 - Accuracy of least square means and standard errors (SD) of different variables in each CLMA category ( $n=162)$

\begin{tabular}{lccccc}
\hline AOLC categórico & $\mathrm{n}$ & EDIFF, mm & EDEV,mm & ADIFF, $\mathrm{cm}^{2}$ & ADEV, $\mathrm{cm}^{2}$ \\
$C L M A$ category & $n$ & $F D I F F, m m$ & $F D E V, m m$ & $R D F F, \mathrm{~cm}^{2}$ & $R D E, \mathrm{~cm}^{2}$ \\
\hline$<50 \mathrm{~cm}^{2}$ & 44 & $0,12 \pm 0,05$ & $0,27 \pm 0,03$ & $0,73^{\mathrm{b}} \pm 0,28$ & $1,35^{\mathrm{c}} \pm 0,17$ \\
$50-55 \mathrm{~cm}^{2}$ & 58 & $0,10 \pm 0,06$ & $0,38 \pm 0,04$ & $-0,29^{\mathrm{c}} \pm 0,15$ & $0,95^{\mathrm{c}} \pm 0,10$ \\
$55-60 \mathrm{~cm}^{2}$ & 34 & $0,29 \pm 0,06$ & $0,36 \pm 0,05$ & $-0,66^{\mathrm{c}} \pm 0,26$ & $1,25^{\mathrm{c}} \pm 0,17$ \\
$>60 \mathrm{~cm}^{2}$ & 26 & $0,17 \pm 0,08$ & $0,38 \pm 0,05$ & $-1,34^{\mathrm{d}} \pm 0,35$ & $1,94^{\mathrm{b}} \pm 0,22$ \\
\hline
\end{tabular}

1 Ver Tabela 1 para descrição das abreviaturas.

1 See Table 1 for abreviations key.

* Médias seguidas de letras distintas na coluna diferem significativamente $(P<0,05)$ pelo teste DMS.

* Means in a column with different superscripts differ $(P<0.001) L S D$ test.

este tecido é facilmente comprimido. Na predição da AOLC, houve tendência de o AEPP se elevar quando a gordura na carcaça aumenta. Entretanto, isto foi verdadeiro até a espessura de gordura de $4 \mathrm{~mm}$.

Na classe de mais de $4 \mathrm{~mm}$, o AEPP diminuiu. Este resultado pode ser explicado pela interação grau de acabamento vs conformação e também pelo fato de que os animais não apresentavam grau de acabamento exagerado nesta classe, com média e desvio-padrão de $5,29 \mathrm{~cm}^{2} \pm 0,15$ (máximo $8 \mathrm{~mm}$ ), assim como pela baixa freqüência de animais na extremidade superior. Esses aspectos podem ser confirmados pelos dados reportados por Greiner et al. (2003), que encontraram menor $\operatorname{EDIFF}(0,38 \mathrm{~mm} \pm 0,15)$ na classe que compreendia EGSC de 5,1 a 7,6 mm, sendo maiores os valores obtidos nas classes extremas de seu estudo.

Quanto à EEPP por categoria de AOLC, os resultados não refletem uma tendência clara. Entretanto, as melhores estimativas da EGSC foram na classe de áreas de músculo menores que $50 \mathrm{~cm}^{2}$. Na estimativa da AOLC, os melhores resultados estão nas classes intermediárias de 50 a $60 \mathrm{~cm}^{2}$. A maior exatidão obtida nesta classe pode ser justificada por sua maior freqüência na população de animais de mesmo tipo biológico, utilizada neste estudo.

Os resultados demonstraram que a magnitude das medidas da carcaça é importante na determinação da exatidão em bovinos de corte, e sugerem uma tendência de medidas extremas de área do músculo Longissimus e da espessura de gordura subcutânea serem menos exatas que medidas intermediárias. Isto sugere que estatísticas para avaliar a exatidão das estimativas das características de carcaça, obtidas pela técnica de ultrasonografia, devam ser geradas a partir de populações representativas de animais dos programas de criação e seleção, da região ou do país em que serão aplicadas.
Tabela 8 - Erros-padrão de predição para espessura de gordura subcutânea (EEPP) e área de músculo (AEPP) para as diferentes categorias de EGS e AOL $(n=162)^{1}$

Table 8 - Standard errors of predicition for subcutaneous fat thickness (FSEP) and for "Longissimus" muscle area (RSEP) for different categories of fat thickness and muscle area $(n=162)^{1}$

\begin{tabular}{lccc}
\hline $\begin{array}{l}\text { Categoria } \\
\text { Category }\end{array}$ & $\begin{array}{c}\mathrm{n} \\
n\end{array}$ & $\begin{array}{c}\text { EEPP, } \mathrm{mm} \\
\text { FSEP, } \mathrm{mm}\end{array}$ & $\begin{array}{c}\text { AEPP, } \mathrm{cm}^{2} \\
\text { RSEP, } \mathrm{cm}^{2}\end{array}$ \\
\hline EGSC & & & \\
$C F A T$ & & & \\
$<2 \mathrm{~mm}$ & 19 & 0,35 & 1,24 \\
$2-3 \mathrm{~mm}$ & 51 & 0,31 & 1,87 \\
$3-4 \mathrm{~mm}$ & 51 & 0,32 & 1,36 \\
$>4 \mathrm{~mm}$ & 41 & 0,45 & \\
AOLC & & & \\
$C L M A$ & & & 1,77 \\
$<50 \mathrm{~cm}^{2}$ & 44 & 0,30 & 1,17 \\
$50-55 \mathrm{~cm}^{2}$ & 58 & 0,46 & 1,48 \\
$55-60 \mathrm{~cm}^{2}$ & 34 & 0,35 & 1,81 \\
$>60 \mathrm{~cm}^{2}$ & 26 & 0,42 & \\
\hline
\end{tabular}

1 Ver tabela 1 para descrição das abreviaturas.

1 See Table 1 for abreviations key.

\section{Conclusões}

A exatidão das medidas ultra-sônicas para a estimativa de características de carcaça obtida neste experimento sugere que os valores dos padrões estatísticos utilizados para medi-las em outros países, como Canadá e Estados Unidos, não são adequados e aplicáveis ao sistema de produção brasileiro.

A direção do viés, a magnitude dos desvios absolutos e o erro-padrão de predição são influenciados pelo tamanho relativo das medidas de área do músculo Longissimus e da espessura de gordura subcutânea na $12^{\mathrm{a}}$ costela da carcaça.

O ultra-som pode ser utilizado com alto grau de exatidão na estimativa do mérito individual de carcaça 
dos animais, auxiliando o produtor e a indústria em decisões acerca da seleção e do manejo para características de composição corporal no animal vivo sem a necessidade de abate.

\section{Literatura Citada}

ANIMAL ULTRASOUND SERVICE - AUS (AUSKey4W). A complete package for image and data analysis designed for use on an IBM/AT or compatible personal computer. Ithaca: Animal Ultrasound Services Inc., 1994. 151p.

BERGEN, R.D.; McKINNON, D.A.; CHRISTENSEN, D.A. et al. Prediction of lean yield in yearling bulls using real-time ultrasound. Canadian Journal of Animal Science, v.76, n.4, p.305-342, 1996.

BRETHOUR, J. R. The repeatability and accuracy of ultrasound in measuring backfat of cattle. Journal of Animal Science, v.70, n.4, p.1039-1044, 1992

BULLOCK, K.D.; BERTRAND, L.L.; BENYSHEK, S.E. et al. Comparison of real-time ultrasound and other live measures to carcass measures as predictors of beef cow energy stores. Journal of Animal Science, v.69, n. 10, p.3908-3916, 1991.

CHARAGU, P.K.; CREWS, D.H. Jr.; KEMP, R.A. et al. Machine effects on accuracy of ultrasonic prediction of backfat and longissimus muscle area in beef bulls, steers and heifers. Canadian Journal of Animal Science, v.80, n.1, p.19-24, 2000.

CREWS JR., D.H.; SHANNON, N.H.; CREWS, R.E. et al. Weaning, yearling, and preeharvestt ultrasound measures of fat and muscle area in steers, bulls, and heifers. Journal of Animal Science, v.80, n.11, p.2817-2824, 2002.

CROSS, H.R. Advances in ultrasound procedures for determining carcass merit in cattle. In: BEEF IMPROVEMENT FEDERATION RESEARCH SYMPOSIUM AND ANNUAL MEETING, 1989, Nashville. Proceedings... Nashville, 1989. p. 1-6.

DUELLO, D.A. The use of real-time ultrasound measurements to predict composition and estimates of genetic parameters of carcass traits in live beef cattle. Ames: Iowa State University, 1993. Thesis (Ph. D. Animal Science, Breeding and Genetics) - Iowa State University, 1993.

GREINER, S.P.; ROUSE, G.H.; WILSON, D.E. et al.The relationship between ultrasound measurements and carcass fat thickness and Longissimus muscle area in beef cattle. Journal of Animal Science, v.81, n.3, p.676-682, 2003.

HASSEN, A.; WILSON, D. E.; WILLHAM, R. L. et al. Evaluation of ultrasound measurements of fat thickness and longissimus muscle area in feedlot cattle: assessment of accuracy and repeatability. Canadian Journal of Animal Science, v.78, n.3, p.277-285, 1998.

HECKMATT, J.Z.; PIER, N.; DUBOWITZ, V. Measurement of quadriceps muscle thickness and subcutaneous tissue in normal children by real-time ultrasound imagining. Journal Clinical Ultrasound, v.16, n.1, p.171-176, 1988.

HERRING, W.; MILLER, D.C.; BERTRAND, J.K. et al. Evaluation of machine, technician, and interpreter effects on ultrasonic measures of back fat and longissimus muscle area in beef cattle. Journal of Animal Science, v.72, n.9, p.2216-2226, 1994.
HOUGHTON, P.L.; TURLINGTON, L.M. Aplication of ultrasound for feeding and finishing animals: a review. Journal of Animal Science, v.70, n.3, p.930-941, 1992.

IOWA STATE UNIVERSITY OF SCIENCE AND TECHNOLOGY. Plastic grid for quick measurement of loin eye (beef). Ames: 1993. AS-234e.

KEMPSTER, A.J.; CUTHBERTSON, A.; HARRINGTON, G. Carcass evaluation in livestock breeding, production and marketing. Great Britain: Granada Publishing, 1982. 306p.

MAY, S.G.; MIES, W.L.; EDWARDS, J.W. et al. Using live estimates and ultrasound measurements to predict beef carcass cutability. Journal of Animal Science, v.78, n.5, p.12551261, 2000.

PERKINS, T.L. The use of real-time, linear-array ultrasound techniques to predict final carcass composition in beef cattle. Lubbock: Texas Tech University, 1992. 187p. Thesis (Ph.D. Animal Science, Breeding and Genetics) - Texas Tech University, 1992

PERKINS, T.L.; GREEN, R.D.; HAMLIN, K.E. et al. Ultrasonic prediction of carcass merit in beef cattle: Evaluation of technician effects on ultrasonic estimates of carcass fat thickness and longissimus muscle area. Journal of Animal Science, v.70, n.9, p.2758-2765, 1992a.

PERKINS, T.L.; GREEN, R.D.; HAMLIN, K.E. Evaluation of Ultrasonic estimates of carcass fat thickness and Longissimus muscle area in beef cattle. Journal of Animal Science, v.70, n.4, p.1002-1010, 1992b.

PERRY, T.C.; AINSLIE, S.J.; TRAXLER, M.J. et al. Use of realtime and attenuation ultrasonic measurements to determine back fat thickness, rib eye area, carcass marbling and yield grade in live cattle. Journal of Animal Science, v.68 (suppl. 1), 337, 1990.

ROBINSON, D.L.; McDONALD, C.A.; HAMMOND, K. et al. Live animal measures of carcass traits by ultrasound: assessment and accuracy of sonographers. Journal of Animal Science, v.70, p.1667-1676, 1992.

ROUSE, G.H.; DUELLO, D.; WILSON, D. et al. The Accuracy of real-time ultrasound scans to measure ribeye area and fat cover on bulls and steers. Beef \& Sheep Research Report, Ames: Iowa State University, 1993. p.15-28.

STATISTICAL ANALYSES SYSTEM - SAS. User's guide. version 8.2. Cary: 2001. 1686p.

SMITH, M.T.; OLTJEN, J.W.; DOLEZAL, H. G. et al. Evaluation of ultrasound for prediction of carcass fat thickness and longissimuss muscle area in feedlot steers. Journal of Animal Science, v.70, n.1, p.29-37, 1992.

STEINER, R.; VOTE, D. J.; BELK, K.E. et al. Accuracy and Repeatability of beef carcass longissimus muscle area measurements. Journal of Animal Science, v.81, n. 8 , p.1980-1988, 2003.

WALLACE, M.A.; STOUFFER, J.R. Predicting beef carcass cut out with ultrasound. Journal of Animal Science, v.39, p.176 (Abstr.), 1974.

WILSON, D.E. Centralized ultrasound processing. Study guides. Atlantic: Iowa State University, 1999. 167p.

Recebido em: 18/01/05

Aceito em: 14/05/05 